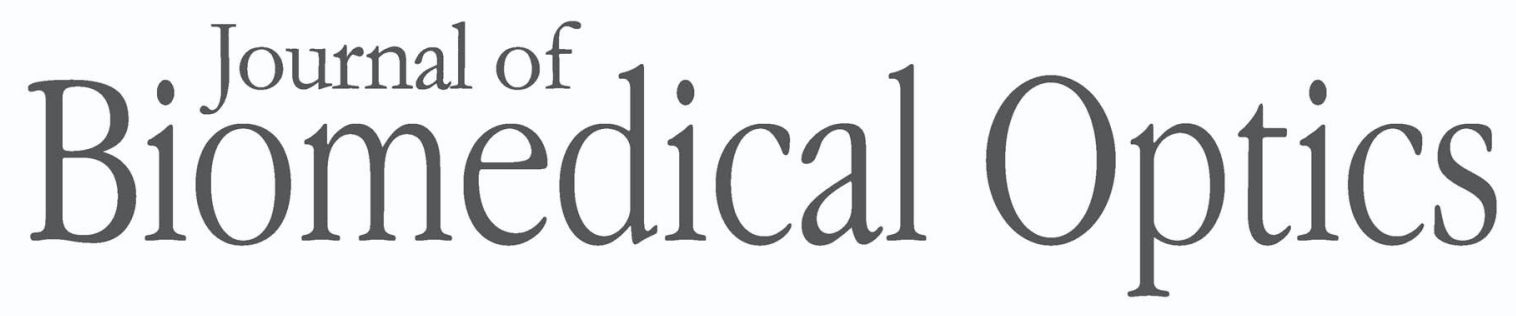

\title{
Follow-up of cortical activity and structure after lesion with laser speckle imaging and magnetic resonance imaging in nonhuman primates
}

Jörn Peuser

Abderraouf Belhaj-Saif

Adjia Hamadjida

Eric Schmidlin

Anne-Dominique Gindrat

Andreas Charles Völker

Pavel Zakharov

Henri-Marcel Hoogewoud

Eric M. Rouiller

Frank Scheffold 


\title{
Follow-up of cortical activity and structure after lesion with laser speckle imaging and magnetic resonance imaging in nonhuman primates
}

\author{
Jörn Peuser, ${ }^{\mathrm{a}, *}$ Abderraouf Belhaj-Saif, ${ }^{\mathrm{b}, *}$ Adjia Hamadjida, ${ }^{\mathrm{b}}$ Eric Schmidlin, ${ }^{\mathrm{b}}$ Anne-Dominique Gindrat, ${ }^{\mathrm{b}}$ \\ Andreas Charles Völker, ${ }^{a}$ Pavel Zakharov, ${ }^{a}$ Henri-Marcel Hoogewoud, ${ }^{c}$ Eric M. Rouiller, ${ }^{b, \dagger}$ and Frank Scheffold ${ }^{a, \dagger}$ \\ a University of Fribourg, Department of Physics, Ch. du Musée 3, CH-1700 Fribourg, Switzerland \\ bUniversity of Fribourg, Department of Medicine, Program in Neurosciences, Ch. du Musée 5, CH-1700 Fribourg, \\ Switzerland \\ 'HFR (Hôpital Fribourgeois), Fribourg, Hôpital cantonal, Department of Radiology, CH-1708 Fribourg, Switzerland
}

\begin{abstract}
The nonhuman primate model is suitable to study mechanisms of functional recovery following lesion of the cerebral cortex (motor cortex), on which therapeutic strategies can be tested. To interpret behavioral data (time course and extent of functional recovery), it is crucial to monitor the properties of the experimental cortical lesion, induced by infusion of the excitotoxin ibotenic acid. In two adult macaque monkeys, ibotenic acid infusions produced a restricted, permanent lesion of the motor cortex. In one monkey, the lesion was monitored over 3.5 weeks, combining laser speckle imaging (LSI) as metabolic readout (cerebral blood flow) and anatomical assessment with magnetic resonance imaging (T2-weighted MRI). The cerebral blood flow, measured online during subsequent injections of the ibotenic acid in the motor cortex, exhibited a dramatic increase, still present after one week, in parallel to a MRI hypersignal. After 3.5 weeks, the cerebral blood flow was strongly reduced (below reference level) and the hypersignal disappeared from the MRI scan, although the lesion was permanent as histologically assessed post-mortem. The MRI data were similar in the second monkey. Our experiments suggest that LSI and MRI, although they reflect different features, vary in parallel during a few weeks following an excitotoxic cortical lesion. 02011 Society of Photo-Optical Instrumentation Engineers (SPIE). [DOI: 10.1117/1.3625287]
\end{abstract}

Keywords: monkey; motor cortex; ibotenic acid; cerebral blood flow; excitotoxic lesion.

Paper 11074RR received Feb. 18, 2011; revised manuscript received Jul. 19, 2011; accepted for publication Jul. 20, 2011; published online Sep. 2, 2011.

\section{Introduction}

The spontaneous capacity of the brain to functionally recover from a lesion is limited, as it is the case after stroke, for instance. A crucial step toward feasible, safe, and efficient clinical application of a therapeutic strategy requires basic investigations and proofs of principle in animal models. The nonhuman primate model is often mandatory to address safety issues and scientific concerns, especially to take into consideration exquisite neural functions present only in primates, ${ }^{1,2}$ such as manual dexterity. ${ }^{3}$ The nonhuman primate model of the macaque monkey was extensively used in our laboratory to assess the extent and mechanisms of spontaneous recovery from spinal cord or motor cortex lesion and to test strategies aimed at enhancing recovery. ${ }^{4-12}$

The nonhuman primate model is suitable to study the consequences of a lesion of the motor cortex, produced by various interventions (surgical lesion, block of a cerebral artery, chemical lesion), ${ }^{11-17}$ and to establish possible mechanisms of recovery. ${ }^{4,14}$ The interpretation of the behavioral data is strongly dependent on the properties of the lesion, such as its extent (volume) and its precise location. Furthermore, the dynamics of the lesion procedure is also likely to influence the magnitude of

*Equal first authorship.

${ }^{\dagger}$ Equal senior authorship.

Address all correspondence to: Eric Rouiller, University of Fribourg, Medicine, Musee 5 - Fribourg, Fribourg 1700 Switzerland. Tel: 412630086 09; Fax: 4126 30096 75; E-mail: Eric.Rouiller@unifr.ch. the deficit and the extent of recovery. In our nonhuman primate model of motor cortex lesion, ${ }^{4,11,12}$ the hand representation in the primary motor cortex (M1) was first delineated with intracortical microstimulation and then damaged by infusion of the excitotoxic ibotenic acid at the most excitable sites, leading to a permanent damage of the corresponding brain area. The precise time course of the devastating effect of ibotenic acid on the cortical tissue is not known, as well as the follow-up of the appearance of the cortical lesion site produced by ibotenic acid infusion in M1 on magnetic resonance imaging (MRI) scans during the weeks post-lesion. To fill this gap, we have implemented in parallel a functional readout [a measure of cerebral blood flow based on laser speckle imaging (LSI) technique] and a structural readout (MRI scan) at consecutive time points post-lesion.

The LSI method ${ }^{18,19}$ is relatively low-cost and easy to implement, and it was broadly used in biomedical studies on small animals over the last decade. ${ }^{20-30}$ The main field of application consisted of creating maps of capillary and perfusion blood flow in tissue. A number of improvements with regard to signal processing and quantitative data analysis have been reported in the literature. ${ }^{31-36}$ The LSI technique can be applied to the exposed cerebral cortex or, in some cases, to the intact (surgically thinned) skull, ${ }^{33,36}$ albeit at reduced performance. Applications for larger animals or humans are impractical due to the limited coherent penetration of light into the skull, although

1083-3668/2011/16(9)/096011/11/\$25.00 C 2011 SPIE 


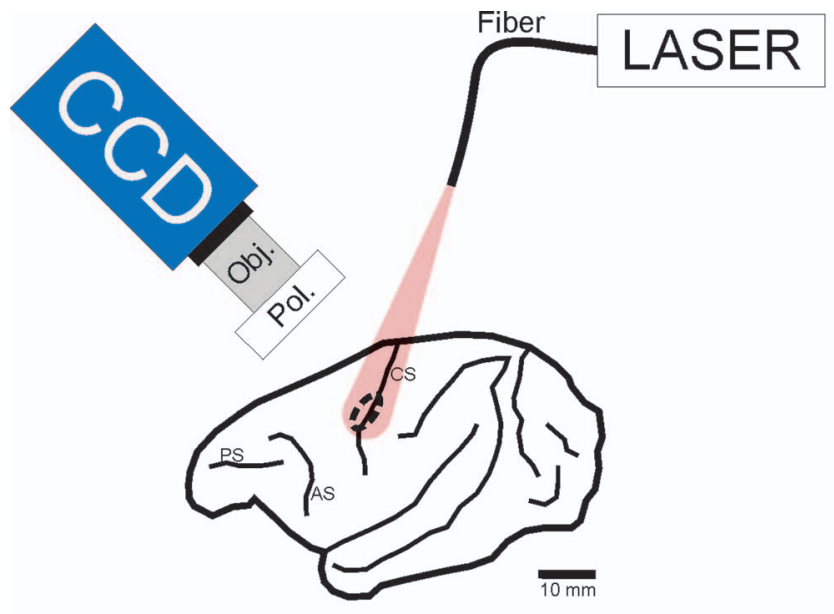

(a)

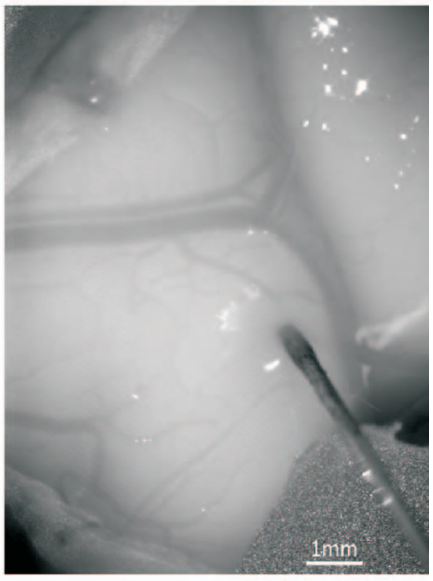

(b)

Fig. 1 (a) Setup of the LSI experiment, schematized over a standard lateral view of the left hemisphere of the macaque monkey. CS $=$ central sulcus; $\mathrm{AS}=$ arcuate sulcus; $\mathrm{PS}=$ principal sulcus. (b) Time integrated image of the cortical surface, taken with the CCD camera. $\mathrm{A}$ vertically oriented blood vessel on the right follows the central sulcus, whereas another blood vessel of large diameter is horizontally oriented over the pre-central gyrus. The image was taken when the needle of an Hamilton syringue was inserted into the motor cortex, rostral to the central sulcus, to perform one of the six penetrations along which ibotenic acid was infused.

intraoperative use of LSI as a monitoring tool in neurosurgery has been reported. ${ }^{37}$

Despite the importance and widespread use of the LSI technique in neurosciences using rodents, a successful application in nonhuman primates is still missing, to our knowledge. The present study thus uses LSI to monitor, in nonhuman primate and over several weeks, the variation of cerebral blood flow of a cortical territory subjected to a permanent lesion induced by infusion of ibotenic acid and, in parallel, the changes of structural properties of the same territory assessed with MRI. A more specific goal was to measure, with LSI, changes of cerebral blood flow during surgery in real time when ibotenic acid was infused in the cerebral cortex to generate a permanent excitotoxic lesion. A further goal was to refine the LSI technique in order to improve the quality of the image by introducing a sliding window processing scheme, as well as to reduce the interference due to movements related to heart beat.

\section{Materials and Methods}

\subsection{Lesion of the Motor Cortex}

LSI and MRI data were collected in parallel from an adult macaque monkey (a male macaca fascicularis: Mk-JH), 7 years old and weighting $7 \mathrm{~kg}$ at the time of the cortical lesion. Additional MRI data were obtained from a second male macaque monkey (Mk-BI; 5 years old, $5 \mathrm{~kg}$ body weight). All procedures were conducted in accordance to the Guide for Care and Use of Laboratory Animals (ISBN 0-309-05377-3; 1996) and approved by local veterinary authorities. As previously reported, ${ }^{11}$ the monkeys were housed in our animal facilities in rooms of $12 \mathrm{~m}^{3}$, in which usually two to four monkeys were free to move and to interact among each other. (A new Swiss regulation was introduced in September 2010 requesting a volume of $45 \mathrm{~m}^{3}$ at least to be given to a group of up to five macaque monkeys.) The monkeys had free access to water and were not food deprived.

To perform the lesion of M1 in both monkeys (Mk-JH and Mk-BI) and, subsequently, to measure cerebral blood flow with
LSI in Mk-JH, the animals were subjected to the following surgical procedures. The monkeys were first tranquilized with ketamine (Ketalar@; Parke-Davis, $5 \mathrm{mg} / \mathrm{kg}$, intramuscularly); atropine was injected $(0.05 \mathrm{mg} / \mathrm{kg}$, intramuscularly) in order to reduce bronchial secretions. Before surgery, the animals were treated with the analgesic Carprofen (Rimadyl $囚, 4 \mathrm{mg} / \mathrm{kg}$, sub-

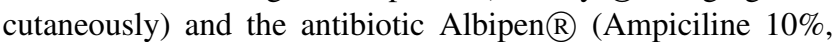
$30 \mathrm{mg} / \mathrm{kg}$, subcutaneously). Subsequently, Mk-JH and Mk-BI were anaesthetized with intravenous perfusion of $1 \%$ propofol (Fresenius $囚$ ) mixed with a 5\% glucose solution (1 volume of propofol and 2 volumes of glucose solution); ketamine was added to the perfusion solution $(65 \mathrm{mg} / 100 \mathrm{ml})$. To prevent edema, Methylprednisolone (Solu-medrol, Pfizer@) was added to the propofol/glucose solution $(1 \mathrm{mg} / \mathrm{ml})$. The level of anesthesia was kept at an optimal level with a perfusion rate of the propofol $/$ glucose mixture of $0.1 \mathrm{ml} / \mathrm{min} / \mathrm{kg}$. All surgeries were performed under sterile conditions. Heart rate, respiration rate, expired $\mathrm{CO}_{2}$, arterial $\mathrm{O}_{2}$ saturation, and rectal temperature were monitored throughout the surgery. After surgery, the monkey received Carprofen (pills of Rimadyl $\AA$ mixed with food) daily

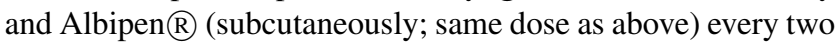
days during one to two weeks.

A squared osseous sector of about $30 \times 30 \mathrm{~mm}$ was opened above the central sulcus, centered at a medio-lateral coordinate (15 $\mathrm{mm}$ from midline) corresponding to the expected position of the hand area in M1. The dura-mater was incised and reclined in order to expose the central sulcus as well as the precentral and postcentral gyri. In monkey Mk-JH, ibotenic acid (Sigma 95\%) was injected at six sites in the precentral gyrus, at a position roughly corresponding to the hand area based on its coordinate and the shape of the central sulcus at this location. The extent of the cortical territory, in which ibotenic acid was injected in $\mathrm{Mk}-\mathrm{JH}$, corresponded to the area of cortex captured by the charge-coupled device (CCD) camera for LSI measurements (Fig. 1). Acquisitions of cerebral blood flow with LSI (see below) were performed before and then after each individual injection of ibotenic acid. A volume of $3 \mu \mathrm{l}$ of 
(a)

(b)

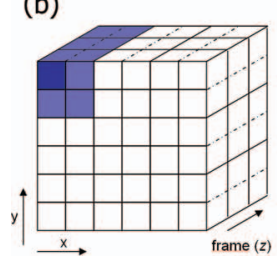

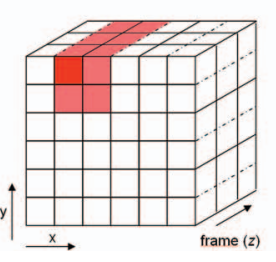
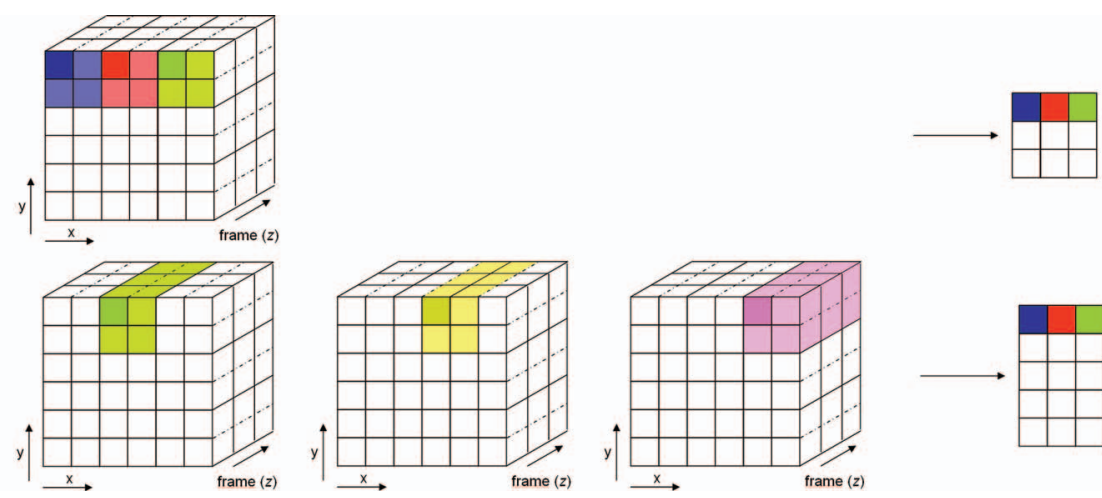

Fig. 2 (a) Standard (temporal) LSI scheme. (b) High resolution LSI introducing a sliding window in space.

ibotenic acid solution (10 $\mu \mathrm{g} / \mu \mathrm{l}$ in phosphate-buffered saline) was injected at each site by using a Hamilton microsyringe, positioned at $2 \mathrm{~mm}$ below the pial surface (Fig. 1). The total volume of ibotenic acid injected in Mk-JH was $18 \mu \mathrm{l}$, an amount representative of volumes injected in monkeys used for behavioral studies. ${ }^{4,11,12}$ In Mk-BI, from which only MRI data were derived, ibotenic acid was injected at 29 sites defined based on intracortical microstimulation, ${ }^{11}$ at which a volume of $1 \mu \mathrm{l}$ was infused (total amount $29 \mu \mathrm{l}$ ). The volume of ibotenic acid injected was larger in Mk-BI, because this animal was involved in the behavioral protocol, ${ }^{12}$ requesting a lesion affecting the entire hand representation in M1. In contrast, Mk-JH was exclusively involved in the present LSI protocol and, therefore, the lesion was adapted to the cortical territory covered by the CCD camera rather than covering the entire hand representation in $\mathrm{M} 1$. In addition, in Mk-JH the infusion sites were somewhat more distant than in Mk-BI, and therefore, the volume injected at each infusion site in $\mathrm{Mk}-\mathrm{JH}$ was larger. At the end of this experimental session aimed at lesioning the motor cortex, the dura mater was put back in place and sutured. The craniotomy was not closed. The muscle and skin were then sutured.

\subsection{Laser Speckle Imaging}

\subsubsection{Data acquisition and image processing}

For each subsequent LSI recording session conducted in Mk-JH only (at 1 and 3.5 weeks post-lesion, also under anesthesia as described above for the initial session), the skin was incised, the muscles reclined, and the dura was reopened as described above. For such a time interval between sessions, there were no adhesions between the dura and the blood vessels. A selected part of the surface of the cerebral cortex was homogeneously illuminated with a $785 \mathrm{~nm}$ single frequency laser (Toptica $\AA$, Munich, Germany), feeding a single mode fiber attached to a collimating lens (Schaefter + Kirchhoff $囚$, Hamburg, Germany; Fig. 1). The whole illuminating device and the CCD camera have been positioned using a stereotaxic frame. The total laser power incident on the brain cortex was about $3 \mathrm{~mW}$ distributed over an area of several $\mathrm{cm}^{2}$, thus preventing any physiological effects or superficial heating of cortical tissue. The diffuse reflected light was monitored in the image plane in the crossed polarization channel with a CCD camera (PCO Pixelfly, $640 \times 480$ pixels, 12 bit, exposure time $12 \mathrm{~ms}$ ). A region of $10 \mathrm{~mm}$ by $7.5 \mathrm{~mm}$ of the cortex was imaged onto the $1 / 2^{\prime \prime} \mathrm{CCD}$ chip at $0.64 \times$ magnification.
Individual measurements of $15 \mathrm{~s}$ duration at 50 frames per second (total 750 frames) have been streamed at full resolution to the hard disk using the Streampix 3 software package (NorPix

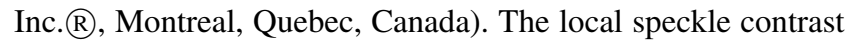
$\mathrm{K}$ was computed from $\mathrm{a} b \times \mathrm{b}$ square of pixels and typically 40 successive frames, ${ }^{24,32,38}$ setting the actual time resolution of the experiment to $0.8 \mathrm{~s}$. The image quality was further improved by implementation of a sliding window average (Figs. 2 and 3), where essentially all camera pixels are replaced by a metapixel (value K). Only a few pixels at the image border need to be excluded from the final image due to the lack of sufficient neighbors. For a raw image with $640 \times 480$ pixels, and typically $\mathrm{b}=5$, this procedure provides a speckle image with a nominal

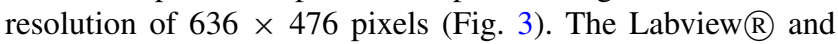

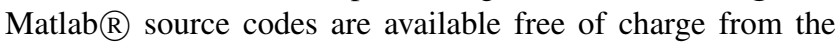
authors (with no support) at http://physics.unifr.ch/en/page/54/.

\subsubsection{Heartbeat filter}

During the experiment the recorded image sequences exhibited a small periodic movement of the cerebral cortex due to the heartbeat/blood pressure variation of the animal. This movement leads to a measurable change in contrast (roughly $+/-10 \%$ ) and in turn slightly blurs the image, when averaging over a sequence of images. To minimize this effect, we have implemented a software filter that allowed us to select images at a certain reference point in the heart beat cycle (Fig. 4). As expected, we found that the contrast time course essentially follows the heart beat with a frequency of approximately 80 beats per minute. A high pass filter was applied to select only the frames of the sequence with an average region of interest (ROI) contrast higher than a specified threshold. All other frames were discarded for the processing of the laser speckle image, thus roughly reducing the number of analyzed raw images by a factor of four to five. As can be seen in Fig. 4, this procedure allows us to reduce the systematic error due to heartbeat from more than 10 to about $3 \%$.

Residual movements of the skull were observed leading to minute shifts in $x$ - $/ y$-directions during a long time recording sequence. It was not straightforward to compensate these movements within our measurement scheme. The presence of these small drifts thus imposes a limit to the total recording time, and therefore, the statistical accuracy of the measurements, in order to avoid blurring effects. Here, we have chosen the image acquisition time such that the influence of these drifts is negligible. 


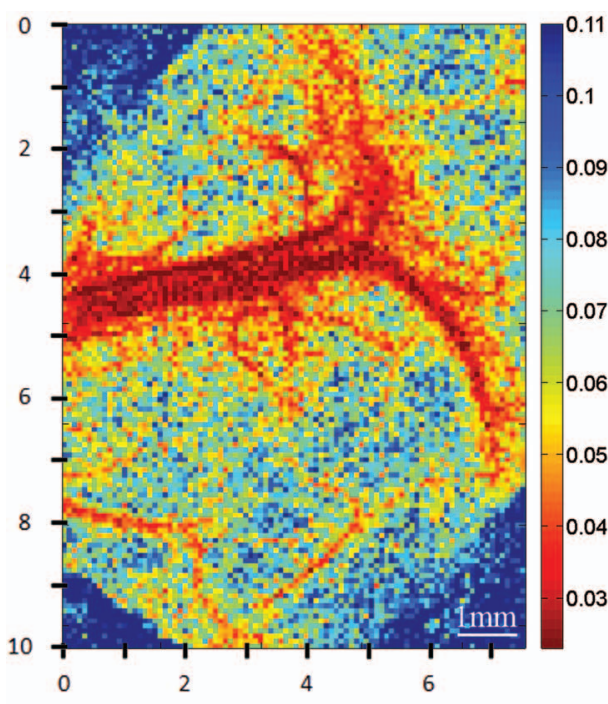

(a)

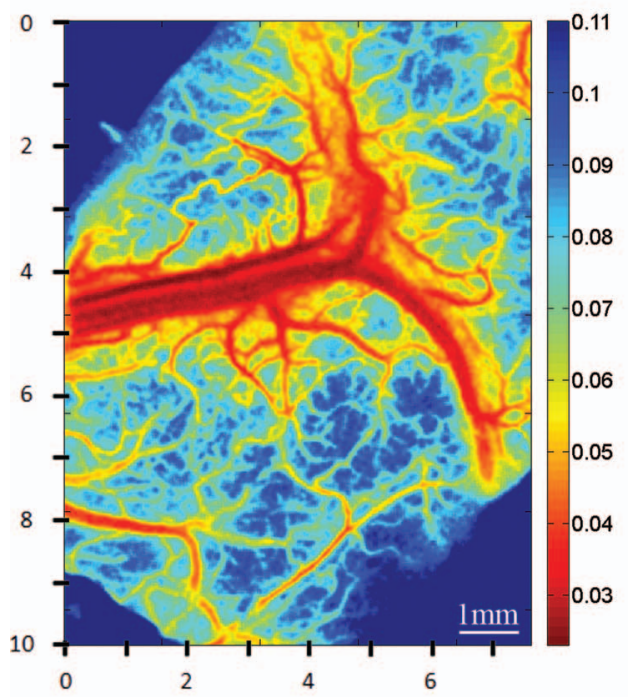

(c)

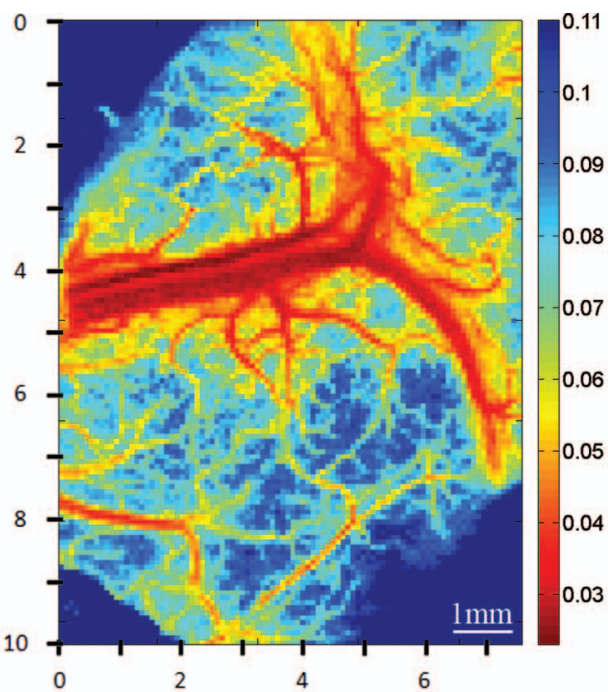

(b)

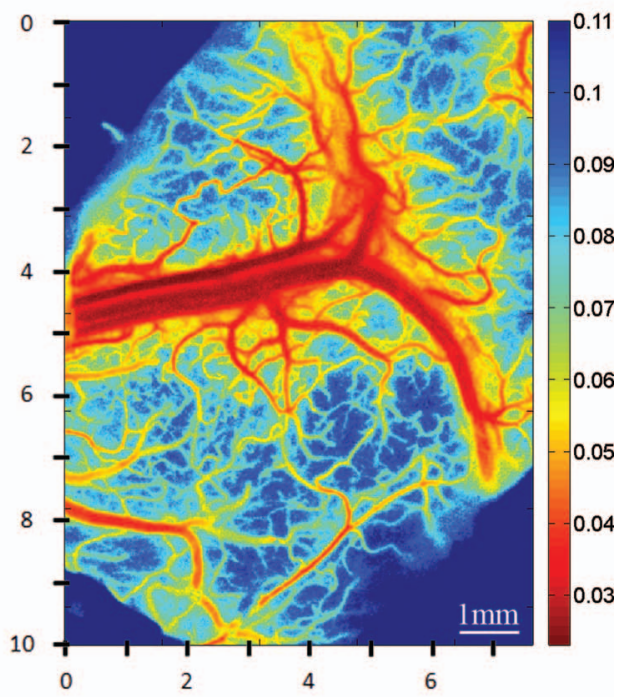

(d)

Fig. 3 Typical LSI in our experiment is constructed from 40 individual frames (resolution $640 \times 480$ pixels) and a spatial average of $5 \times 5$ camera pixels is performed at each point resolution in a final image with a nominal resolution of $636 \times 476$ pixels. Laser speckle images of the cerebral motor cortex calculated with different parameter settings: spatial averaging box size is set to $5^{2}$ pixels for (a), (b), and (c); for (d) it is $2^{2}$ pixels. (a) No time average, single frame, standard resolution scheme. (b) Time averaging over 40 frames, standard resolution scheme. (c) Time averaging over 40 frames, high resolution scheme (sliding window). (d) Time averaging over 250 frames, high resolution scheme (sliding window). The number of raw pixels used for the calculation of one LSI metapixels is $1000 \mathrm{for}(\mathrm{b})$, (c), and (d) (example: box size $*$ frames $=5^{2}$ pixels $* 40$ frames $=1000$ pixels). Axes labeling: $x-y$ display the camera pixel at 0.64 magnification. The speckle contrast is color coded as shown by the color bar on the right of each LSI.

\subsection{Magnetic Resonance Imaging Acquisition}

The MRI data were acquired in Mk-JH and Mk-BI using a 1.5 Tesla Siemens $\odot$ Symphony magnetic resonance scanner. To allow direct comparison of the extent of the cortical lesion at regular time points after the lesion, the anesthetized animal was placed in a decubitus ventral position with his head stabilized into a nonferromagnetic fixation frame with ear, mouth, and eye bars. MRI was performed under heavy sedation induced with subcutaneous injections of ketamine (Graeub $囚 10 \mathrm{mg} / \mathrm{kg}$ ) associated with medetomidine (Graeub $\AA 1 \mathrm{mg} / \mathrm{kg}$ ). At the end of the acquisition, the anesthesia was reversed with Atipamezol (Pfizerß, $0.25 \mathrm{mg} / \mathrm{kg}$ ). The acquisition parameters of the MRI data were the following: total of 19 images, slice thickness $2 \mathrm{~mm}$,
2 TSE (TR $4500 \mathrm{~ms}$ and TE $129 \mathrm{~ms}$ ), field of view $107 \mathrm{~mm}$ $\times 140 \mathrm{~mm}$.

\subsection{Necropsy and Histology}

At the end of the experiment, the monkeys were sacrificed ${ }^{5,7}$ for histological analysis of the lesion. Mk-JH was sacrificed a few days after the last LSI session, whereas Mk-BI was sacrificed several months post-lesion as it was involved in a study of functional recovery. ${ }^{12}$ The monkeys were sacrificed under deep anesthesia [initiated first with an i.m. ketamine injection followed by an i.p. lethal dose of sodium pentobarbital $(90 \mathrm{mg} / \mathrm{kg})]$ by transcardiac perfusion with $0.9 \%$ saline $(400 \mathrm{ml})$ continued with fixative (three liters of four paraformaldehyde in $0.1 \mathrm{M}$ phosphate 


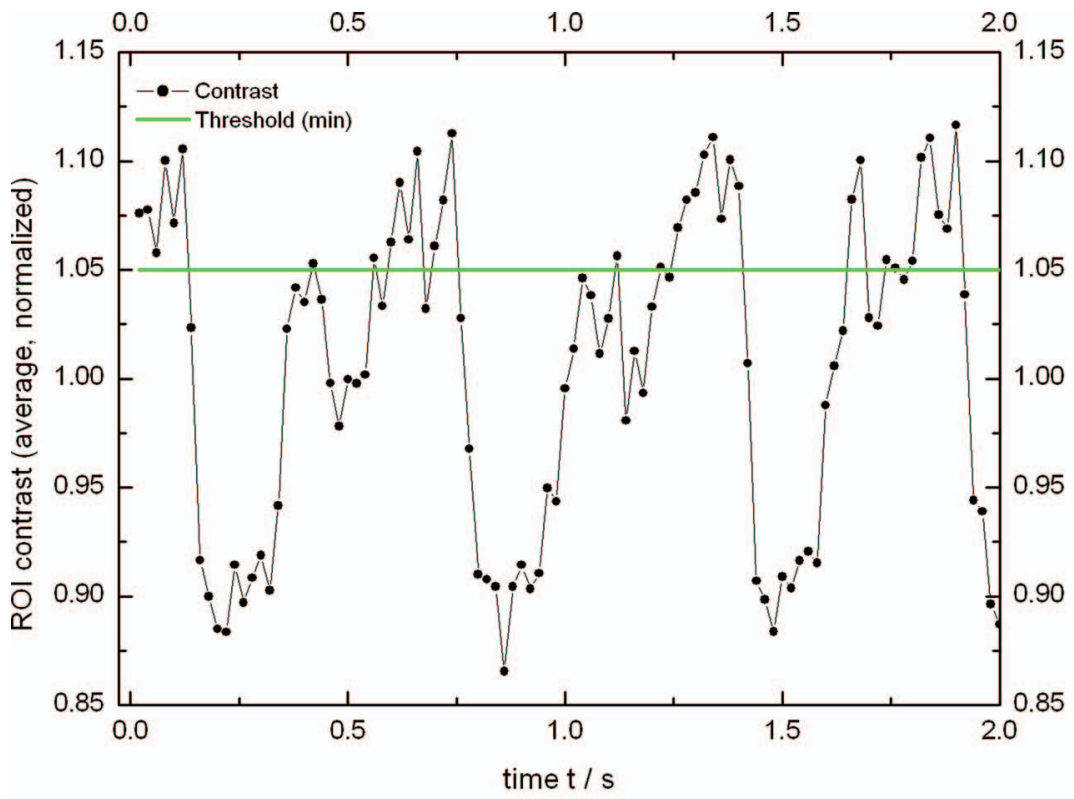

(a)

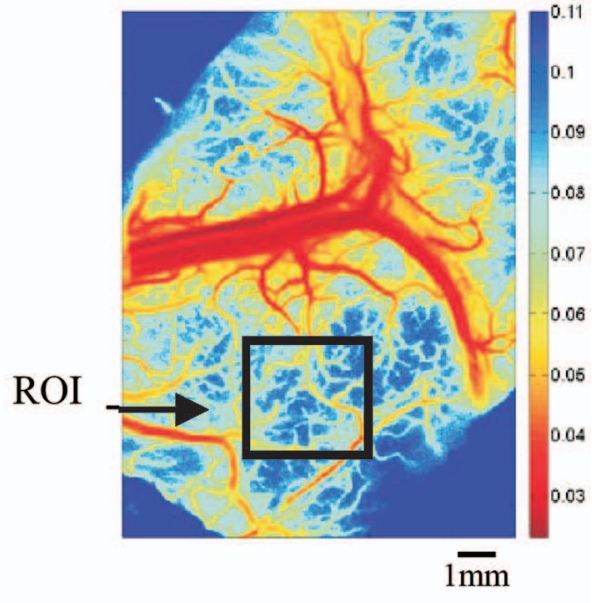

(b)

Fig. 4 (a) Heart beat signal computed from the average contrast in a ROI of $100 \times 100$ pixels from successive individual frames. The data were normalized and baseline corrected. The green line shows the minimum threshold, all other frames below that line will not be used to calculate a high resolution contrast image. (b) LSIs of the cerebral motor cortex calculated from $5^{2}$ pixels and 40 successive frames. The ROI analyzed for the heartbeat correction is indicated by the black square.

buffer, $p \mathrm{H}=7.6$ ) and solutions (two liters each) of the same fixative containing increasing concentrations of sucrose $(10,20$, and $30 \%)$. The brain was removed, dissected, and stored in a sucrose solution $(30 \%)$ for 1.5 to 2 weeks. Frozen sections of the brain were then cut in the frontal plane at a thickness of $50 \mu \mathrm{m}$. Eight series of sections were collected with a cryotome (HM560, MICROM $®$, Switzerland). Among these series, one was Nisslstained and one was immunocytochemically treated (SMI-32 antibody against a non-phosphorylated neurofilament epitope), as previously reported. ${ }^{7,39}$ Furthermore, additional series were processed for other markers, such as Neuronal Nuclei [(NeuN) neuronal marker] and glial fibrillary acidic protein [(GFAP) glial marker], following previously described protocols. ${ }^{40}$

\section{Results}

\subsection{Injections of Ibotenic Acid}

In the first LSI recording session conducted in Mk-JH, LSI acquisition was immediately made after incision of the dura in order to establish the cerebral blood flow level corresponding to the reference state under stable propofol anesthesia. Then, this first session comprised the procedure of ibotenic acid infusion in the motor cortex in order to produce a permanent cortical lesion, comparable to lesions made in the course of previous studies in our laboratory. ${ }^{4,11,12}$ Six penetrations with the needle of a Hamilton syringe were performed (Fig. 5), in the part of cerebral cortex rostral to the central sulcus, corresponding to M1.

\subsection{Speckle Contrast Imaging (Laser Speckle Imaging)}

The LSI in the reference state exhibited a high contrast [Figs. 6(a) and 7], corresponding to a moderate blood flow. As expected, the infusion of ibotenic acid at the first site of injection (Fig. 5) produced an immediate decrease of contrast, in line with an increase of cerebral blood flow (Fig. 7). Note, however, that the contrast slightly increased in the few minutes following the infusion of ibotenic acid at one site, as shown by the three LSI data points taken at 1.5 min intervals following the first post-infusion LSI acquisition. The same time course of contrast change was observed at all six injection sites during the few minutes following the actual infusion [Fig. 7(a)]. The next infusion at sites \#2 and \#3 produced a further decrease of contrast (increase of blood flow) [Fig. 7(a)]. As of the fourth injection site, the contrast reached a stable lowest level (maximal blood flow), although the small rebound of contrast was still

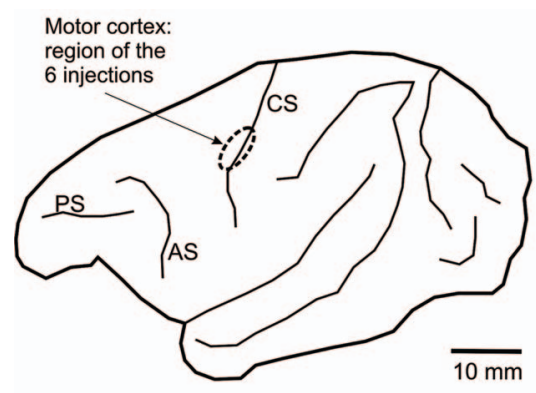

(a)

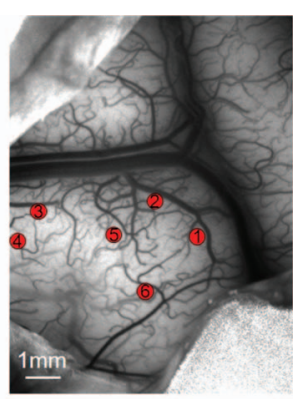

(b)
Fig. 5 (a) Lateral view of the left hemisphere of $\mathrm{Mk}-\mathrm{JH}$, showing the cortical territory (circle) in which a lesion of the motor cortex was performed with infusion of ibotenic acid along six penetrations in the pre-central gyrus. The ROI is presumably located in the zone corresponding to the hand area. (b) CCD image (slightly tilted to the left) of the cortical surface in the pre-central gyrus, with location on the cortical surface of the six syringe penetrations (one to six). Same orientation as in the right panel of Fig. 1 . 
(a)

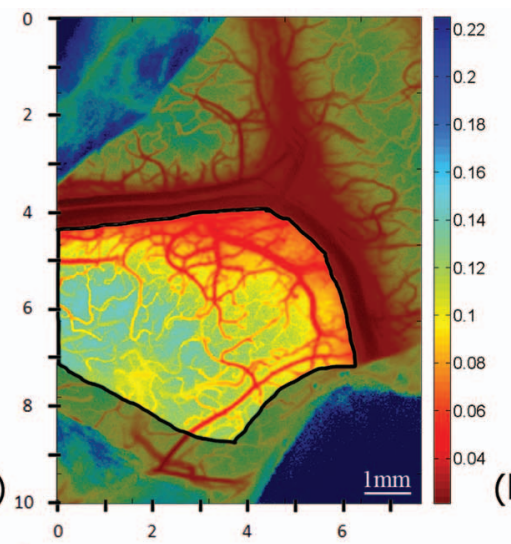

(c)

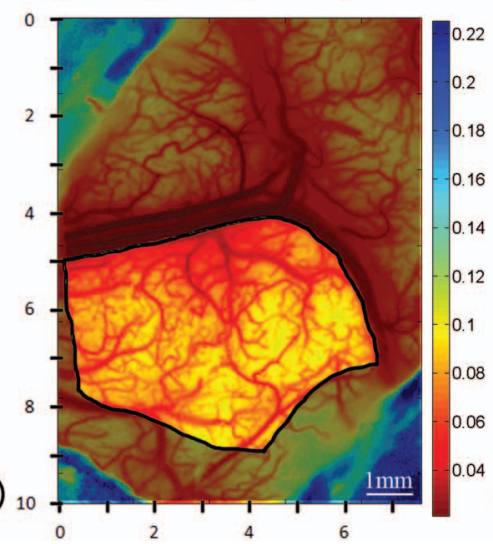

(b)

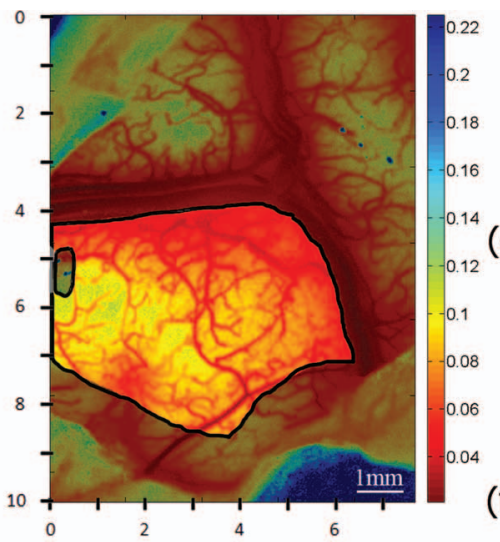

(e)
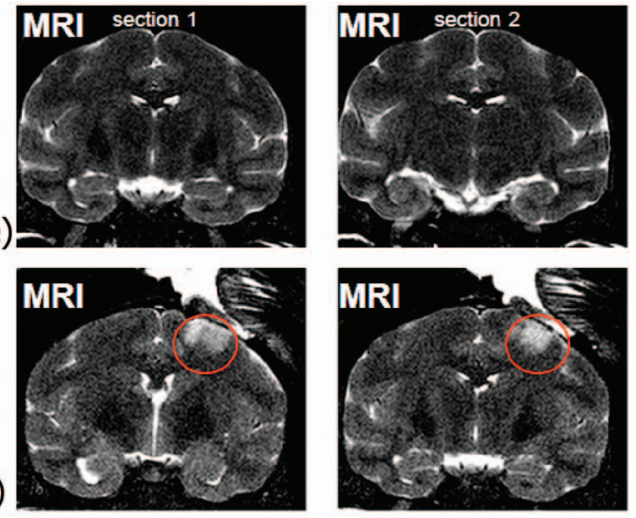

(d)

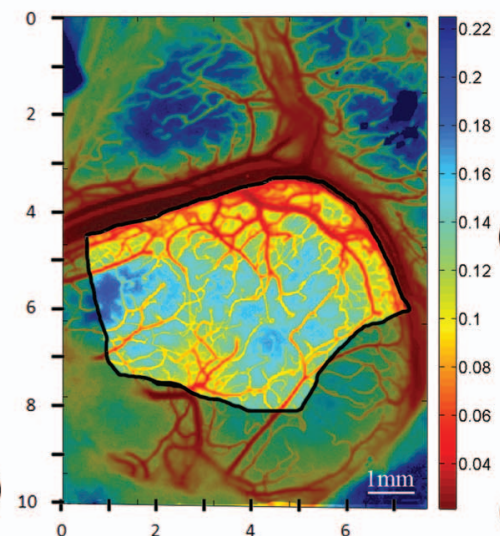

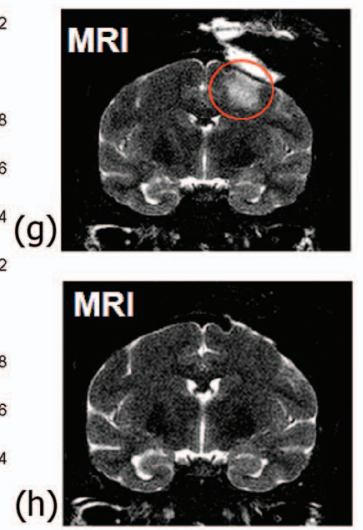
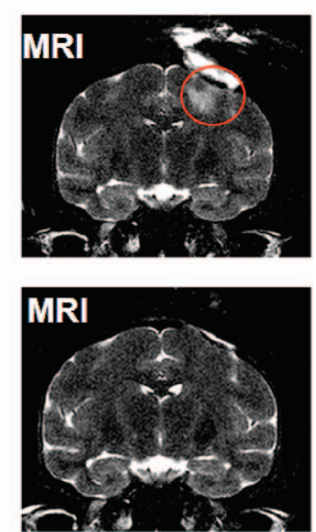

Fig. 6 LSI $[(a),(b),(c),(d)]$ and MRI $[(e),(f),(g),(h)]$ are compared in Mk-JH within a time frame of 3.5 weeks; (a), (e) reference before the injections; (b), (f) LSI taken 60 min post-lesion, MRI at one day post-lesion; (c), (g) LSI and MRI at one week post-lesion (one day apart from each other); (d), (h) LSI and MRI 3.5 weeks post-lesion (one day apart from each other). The highlighted area in the LSI figures shows the ROI wherein the average contrast was calculated for the quantitative data shown in Fig. 7 . Calculation parameters for the LSI: 250 frames out of $750, \mathrm{HBC}$ filter, sliding box, box $=52$ pixels. The left and right MRI images on the same horizontal panel are taken at two rostro-caudal levels, distant by 2 mm, intercepting the ROI in the motor cortex.

present in the few minutes following each individual injection [Fig. 7(a)]. LSI was acquired during 25 more minutes after injection at the last site (site \#6), taken at roughly 5 min intervals: there was a slow increase of contrast, finally reaching a stable level after about 75 min, situated in between those observed after the second and third injections of ibotenic acid. This contrast level at plateau is illustrated for the ROI in Fig. 6(b), showing that it was clearly diminished as compared to the reference state [Fig. 6(a)].

At that step, the dura was sutured (together with muscles and skin), the anesthesia was discontinued and the monkey was returned to the animal room. LSI was reacquired one week later and the contrast level was comparable to that observed about one hour after lesion [Figs. 6(c) and 7(b)]. The next LSI data point was acquired 3.5 weeks post-lesion, showing a dramatic increase of contrast, reaching a value higher than the initial reference value [Figs. 6(d) and 7(b)]. These data are indicative of a substantial decrease of blood flow at 3.5 weeks post-lesion, in line with the notion that ibotenic acid infusion produces a permanent lesion of the cortical tissue.

\subsection{Comparison of Laser Speckle Imaging, Magnetic Resonance Imaging and Histology}

LSI and MRI were not acquired the very same day as the two facilities are located at distant sites. The first MRI image [Fig. 6(e)] was taken two days before the first LSI recording acquisition [Fig. 6(a)], both representing the reference state pre-lesion. The second MRI image was acquired one day after the first LSI session during which the ibotenic acid was infused at six sites. The zone of infusion corresponds to a marked hypersignal [red circle in Fig. 6(f)] present in the pre-central gyrus. The hypersignal in the MRI was found to be maintained in the same area [red circle in Fig. 6(g)] one week later, in parallel to the decrease of contrast in the LSI [Figs. 6(c) and 7(b)], associated to an increase of cerebral blood flow. The last MRI image was taken 3.5 weeks post-lesion [Fig. 6(h)], showing a complete disappearance of the hypersignal, in parallel to the dramatic decrease of cerebral blood flow at the same time point, reflected by the increase of LSI contrast [Figs. 6(d) and 7(b)]. The LSI and MRI signals in the ROI follow a parallel time course, at least for the four time points considered in the present study (reference, briefly after lesion, one week post-lesion, and 3.5 days post-lesion). The lesion generated by ibotenic acid infusion in the motor cortex is visible with both LSI and MRI only during a few days (at least one week). Later on, after 3.5 weeks, the signals in both LSI and MRI returned in the direction of the reference appearance prelesion [compare Figs. 6(a) and 6(d) for LSI; Figs. 6(e) and 6(h) for MRI], although the LSI signal had a higher contrast, corresponding to a decrease of cerebral blood flow. Evidence for the presence of a permanent cortical lesion was provided by the histology post-mortem for Mk-JH (Fig. 8). As shown in SMI-32 


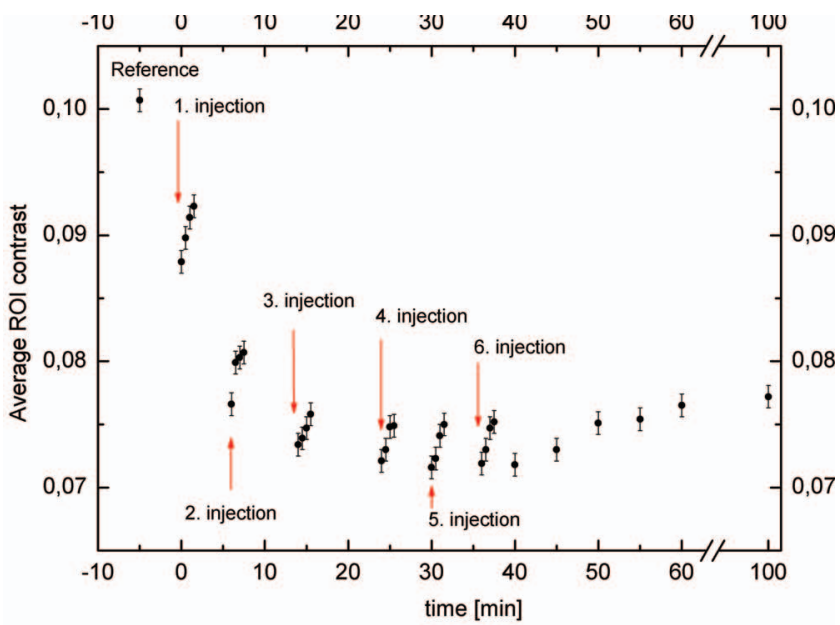

(a)

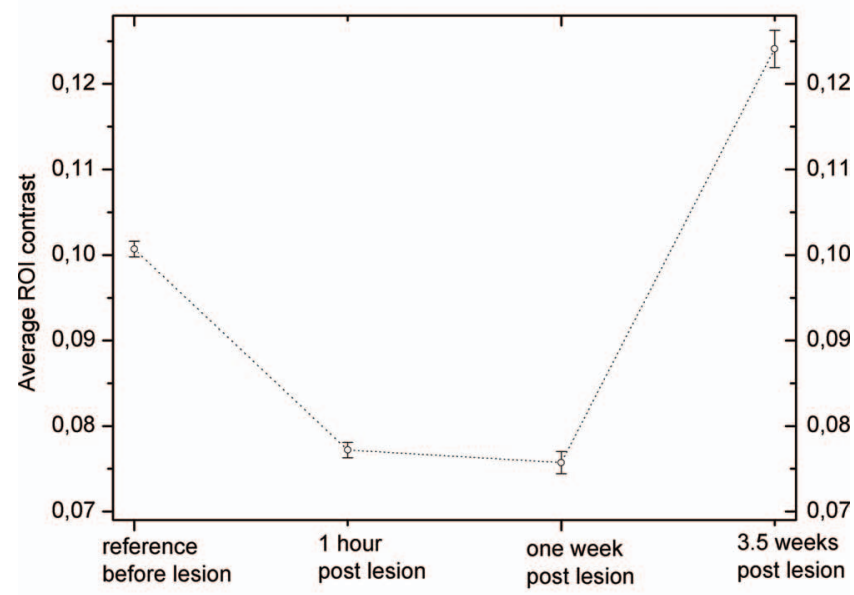

(b)

Fig. 7 Time course of maximum, minimum, and average ROI contrast on (a) the day of ibotenic acid injection in Mk-JH as well as (b) at two further distant time points. In (a), the data points related to the six infusions of ibotenic acid were acquired after offset of the injection (the needle was removed from the tissue). In (b), the data point at reference and at one hour post-lesion corresponds to the data point at reference and at $100 \mathrm{~min}$ in the upper panel (errors bars were estimated from the time course of the measurements). The data at one and 3.5 weeks were derived from six and five independent measurements, respectively.

stained material (Fig. 8), the infusion of ibotenic acid produced a clear disruption of the cortical layers in the lesion site (delineated with the dashed line) without SMI-32 labeled pyramidal cells, whereas in the intact tissue adjacent to the lesion territory the cortical layers III and V are clearly visible by the presence of pyramidal neurons stained with SMI-32 (panels A1 and A2 in Fig. 8 at higher magnification). The appearance of the lesion is similar to that previously shown in monkeys subjected to such lesion and involved in our functional recovery protocols. ${ }^{11}$ The focal cortical lesion does not generate a cavity in the gray matter but, as seen on Nissl staining, it corresponds to a homogeneous territory with loss of neuronal cell bodies in all cortical layers (Fig. 8, panels B1 and B2; comparable to a previous description in the rat: see Fig. 1 in Leroux et al. ${ }^{41}$ ). Furthermore, the lesioned territory seen with the above two markers (SMI-32 and Nissl) matches the zone corresponding to an interruption of the

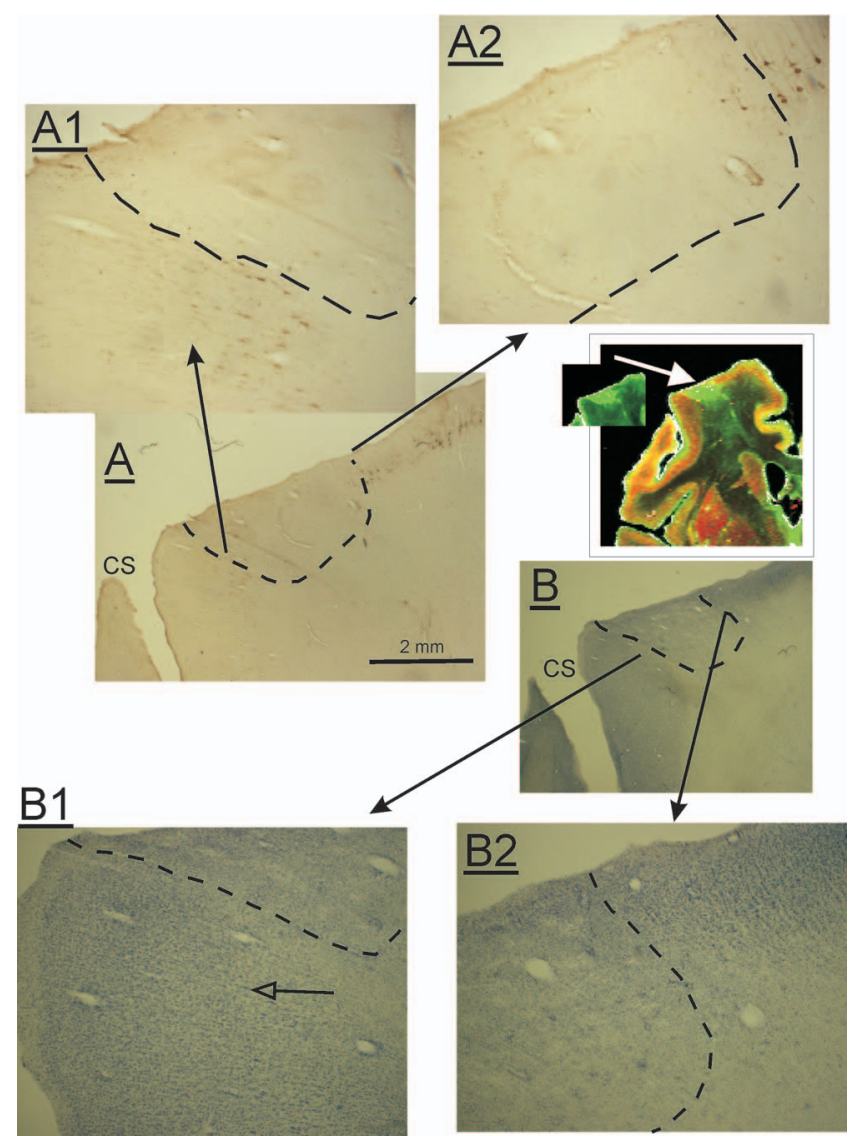

Fig. 8 Histological assessment of the permanent lesion in the motor cortex in $\mathrm{Mk}-\mathrm{JH}$, on a frontal section of the motor cortex. Panel $\mathrm{A}$ is a low magnification of the lesion area, as seen with the marker SMI-32. In the top row, the panels $\mathrm{A} 1$ and $\mathrm{A} 2$ are higher magnification of the lateral and medial edges of the lesion territory, respectively, also in SMI-32 material. The damaged territory corresponds to an interruption of the layers III and V pyramidal neurons stained with SMI-32. In the middle, panel $B$ is a low magnification in Nissl material of the lesion area. The panels B1 and B2 in the bottom row show at higher magnification the edges of the lesion with Nissl staining. In all panels, the dashed line delimits the lesion territory. The open arrow in panel B1 points to large Nissl stained neurons in layer $\mathrm{V}$ in an intact territory located slightly more lateral than the lesion. The inset in the middle at the right is a low magnification view of the lesion (white arrow), as seen in a combined NeuN (red) and GFAP (green) stained material. The corresponding lesion area is shown for GFAP labeling alone (in green), next to the white arrow. CS = central sulcus.

neuronal marker NeuN and, in contrast, an increase of the glial marker GFAP (Fig. 8, middle inset). The MRI data obtained in Mk-BI (not shown) exhibit a similar time course of the hypersignal associated to the ibotenic acid lesion in M1, as illustrated for Mk-JH in Fig. 6.

\subsection{Comparison of the Lesion Size Assessed with Magnetic Resonance Imaging, or Post-mortem on Histological Sections or with Laser Speckle Imaging}

Based on consecutive histological sections as the one illustrated in Fig. 8 and using an ad-hoc function of the Neurolucida software (based on the Cavalieri method) as previously described, ${ }^{11}$ the volume of the histological permanent lesion affecting the 
gray matter was estimated to be $18.8 \mathrm{~mm}^{3}$ in $\mathrm{Mk}-\mathrm{JH}$ (after sacrifice, one month post-lesion). For comparison, the volume of the lesion as reflected by the hypersignal in the MRI scan (computed with the software OsiriX $\left(\right.$ ) was $436 \mathrm{~mm}^{3}$ at one day post-lesion, $405 \mathrm{~mm}^{3}$ after one week, and $341 \mathrm{~mm}^{3}$ after two weeks. After 3.5 weeks, the lesion was no longer visible on the MRI scan [Fig. 6(h)]. In the second monkey (Mk-BI), the volume of the lesion assessed based on the hypersignal on the MRI scan was $262 \mathrm{~mm}^{3}$ one day post-lesion, $146 \mathrm{~mm}^{3}$ one week after lesion, $135 \mathrm{~mm}^{3}$ two weeks after lesion, and $97 \mathrm{~mm}^{3}$ three weeks after the lesion, whereas, 10 months post-lesion, the volume of the permanent lesion was estimated at $20.1 \mathrm{~mm}^{3}$ on SMI-32 stained histological material.

As expected, mostly due to edema in the days following the cortical lesion, the volume of the lesion derived from the MRI scans is clearly larger than the final volume histologically determined, by a factor of about 10 to 20 . The difference may also comprise a deviation between the two methods to measure the volume of the lesion: although the infusion was aimed to the gray matter, some spread to the white matter is likely, which may be detected on the MRI scans, whereas the histological assessment was limited to the gray matter.

Finally, for comparison, the volume of the lesion was tentatively calculated from the LSI data at the time point at which a territory with reduced blood flow was observed, corresponding to the lesion. Based on an estimation of the surface of the cortical territory with reduced cerebral blood flow as seen after 3.5 weeks in Mk-JH [Fig. 6(d)] and considering an average cortical thickness of $2 \mathrm{~mm}$, the volume of the cortical lesion as derived from LSI data is estimated to be around $58 \mathrm{~mm}^{3}$. Again, this figure is larger than the actual volume of the lesion histologically determined, but only by a factor of two to three in the comparison between LSI after 3.5 weeks and histology after four weeks. As the LSI approach does not permit an assessment of the cortical thickness in which cerebral blood flow is modified, one may compare in Mk-JH the extent of cortical surface exhibiting an increase of cerebral blood flow detected with LSI during the excitotoxic phase (immediately after infusion of ibotenic acid and one week later) with the cortical surface of the hypersignal observed from MRI one day and one week post-lesion. The cortical surface of the MRI hypersignal was $78.3 \mathrm{~mm}^{2}$ at one day and $71.7 \mathrm{~mm}^{2}$ at one week post-lesion, whereas the zone of increased blood flow corresponded to a cortical surface estimated at $22 \mathrm{~mm}^{2}$, both on the day of ibotenic acid infusion and one week later.

\section{Discussion}

The present study, based on a parallel assessment of a cortical lesion using LSI and MRI in nonhuman primates, provides evidence that LSI and MRI are tools suitable to monitor the time course of a cortical lesion, for subsequent correlation with the behavioral recovery curve, at least in the present experimental conditions (monkey, ibotenic acid cortical lesion). Although the time course of LSI and MRI signals change in parallel in a time window of a few weeks post-lesion, there is no evidence for a direct relation between LSI and MRI signals, as the former measures cerebral blood flow whereas the latter reflects mostly edema (see below) and tissue infarct - degeneration. The real time LSI data during the surgery show that, when ibotenic acid is infused in the cerebral cortex at multiple adjacent sites in a bit less than an hour, there is an immediate increase of cerebral blood flow, which is still present after one week (Fig. 7). As far as the extent of the cortical lesion is concerned, the present study provides estimates of the ratio between the sizes of the lesion derived from LSI data and from MRI data during the few weeks following the damage, as well as with the size of the corresponding permanent lesion histologically assessed.

Moreover, the LSI and MRI data may contribute to a better understanding of mechanisms underlying functional recovery after cerebral cortex lesion, such as collateral blood or reperfusion after ischemic stroke. ${ }^{28,30}$ The LSI method may also be suitable to assess whether cortical areas adjacent to the lesion change their activity, as they may contribute to the recovery, as shown in a rat model of M1 lesion. ${ }^{26}$ The improvements of the LSI analysis method [heart beat compensation (HBC) filter, high resolution and low noise LSI] have successfully been applied to the present data set, revealing detailed and additional information on cerebral blood flow that are not visible in the MRI. The LSI adapted technique permitted here to monitor online the cerebral blood flow while ibotenic acid was infused in the cerebral cortex of macaque monkey to produce a permanent lesion. At the onset of ibotenic acid infusion, the cerebral blood flow dramatically increased almost immediately, followed by a slight reversal during the next five minutes (Fig. 7).

Follow-up studies are available in human subjects after stroke, during time windows ranging from $12 \mathrm{~h},{ }^{42}$ one month, ${ }^{43}$ and up to four months. ${ }^{44}$ MRI is a standard tool used in the clinical evaluation of acute stroke, mainly based on the weighteddiffusion imaging setting, offering the best sensitivity to assess acute ischemic lesions for instance. ${ }^{45,46}$ In the present study, the T2-weighted imaging setting was chosen as it provides better sensitivity and resolution to detect a chronic cortical lesion and, therefore, is better suited to conduct a longitudinal study over several weeks. Nevertheless, the T2 setting allowed detection of the ibotenic acid lesion in Mk-JH and in Mk-BI already relatively early, about $24 \mathrm{~h}$ post-lesion. This observation contrasts with previous $\mathrm{T} 2$ data in humans after stroke, exhibiting no hypersignal either immediately post-infarct or after $24 \mathrm{~h}^{43}$ This discrepancy may be explained by the different type of lesion, stroke in humans, and ibotenic acid in the macaque monkeys Mk-JH and Mk-BI. More consistent is the situation observed after about a week, with a hypersignal detected with $\mathrm{T} 2$ in both humans ${ }^{43}$ and monkeys Mk-JH and Mk-BI (present study). After three to four weeks, there was again a discrepancy, as the $\mathrm{T} 2$ hypersignal was present in human subjects ${ }^{43}$ but no longer in Mk-JH and Mk-BI. In human subjects after stroke, a T2 hypersignal was still present after four months. ${ }^{44}$ However, the latter authors reported that the volume of the infarct lesion in humans significantly diminished in the chronic phase, possibly due to lesion consolidation-related changes. In Mk-JH and MkBI, similar changes may have happened, but even more rapidly in the present case of a much smaller ibotenic acid lesion, corresponding to a total disappearance of the lesion after 3.5 weeks in MRI. In macaque monkeys, in contrast to the present study, an ibotenic acid lesion in the hippocampus remained visible about 4 years later, ${ }^{47}$ but the lesion was performed at the neonatal stage (12 to 16 days after birth), whereas in Mk-JH and Mk-BI the lesion took place at the adult stage, thus possibly accounting for this discrepancy (at least in part). After lesion of the hippocam- 
pal formation in adult macaques with ibotenic acid infusion, ${ }^{48}$ a prominent hypersignal was found in T2-weighted images after one week post-lesion (as is the case in the present study), but the hypersignal then weakened at two weeks post-lesion, before it gradually disappeared. The optimal time for post-lesion scan was thus identified as less than two weeks. ${ }^{48}$

The time course of the neurotoxic lesion observed here in the nonhuman primate is generally consistent with that reported from MRI data for the same type of lesion in the rat, either in the cerebral cortex ${ }^{49}$ or in the striatum, ${ }^{50,51}$ namely a hypersignal during a few days, followed then by a progressive decrease after one to two weeks. From the time course of the cortical lesion in the rat derived from MRI ${ }^{49}$ it was concluded that functional and behavioral investigations should be initiated about two weeks after the neurotoxic lesion to avoid transient confounding factors.

As far as cortical lesion produced by ibotenic acid injection in monkeys is concerned, $4,11,12,16,52-54$ the present parallel LSI and MRI study extends previous information in the time dimension. As a result of ibotenic acid infusion, the excitotoxic increase of activity, as reflected by an increase of cerebral blood flow visualized with LSI, does not last only a few hours but it is still present after at least a week. The clinical consequence of the ibotenic acid infusion in M1 in monkeys has a very rapid time course as the flaccid paralysis of the contralateral hand occurs already 10 to 15 min after the infusion of ibotenic acid. ${ }^{4}$ Based on the parallel time course of both LSI and MRI, the present data suggest that the MRI hypersignal is possibly associated, at least in part, to the excitotoxic activity of the ibotenic acid injections. After 3.5 weeks, the excitotoxic activity has disappeared in both LSI and MRI. The disappearance of the hypersignal in T2-weighted images could be correlated with the decrease of the cytotoxic edema, as previously reported in the rat. ${ }^{49}$ This observation would then be in line with the time course reported after ibotenic acid injection in the hippocampal formation, ${ }^{48}$ as well as with excitotoxic lesion in rats. ${ }^{49-51}$ It remains to be determined at which precise time point (between one and 3.5 weeks) the excitotoxic activity disappears, to turn into a cortical region with diminished cerebral blood flow, as compared to the pre-lesion reference level. However, as previously claimed based on ibotenic acid lesion in the hippocampus of monkeys, the edema associated to the lesion may, to a large extent, contribute to the hypersignal in the MRI imaging during a few weeks post-lesion. ${ }^{48}$ Interestingly, the same authors found that T2-weighted imaging obtained after one to two weeks post-lesion was an accurate predictor of the extent of the lesion determined a year later from post-mortem histology. In a study on macaque monkeys subjected to ibotenic acid lesion of the cortical areas MT (middle temporal) and MST (medial superior temporal) ${ }^{54}$ the hypersignal in T2-weighted MRI imaging associated to the lesion remained visible after several months. However, the lesions were much larger than the present lesions in M1, as the total amount of ibotenic acid injected in MT and MST were about five to eight times bigger.

LSI was previously used in rats (ministroke models) to monitor the changes of cerebral flow at the surface of the cerebral cortex during and after blood vessels occlusion, ${ }^{25-29}$ as well as the reperfusion after reversal of vascular ligation. ${ }^{28}$ In these studies, the blood flow was measured with LSI during minutes and hours after the lesion, and up to $24 \mathrm{~h}$ in the reperfusion model. ${ }^{28}$
Besides the very different type of lesion, ibotenic acid initially provoking (for at least a week) an increase of cerebral blood flow reflecting over-excitation, whereas vessels' occlusion generates a dramatic decrease of perfusion, the present study provides LSI measurements at much more distant time points from the lesion (one and 3.5 weeks). The permanent lesional property, characterized by a reduced cerebral blood flow, is established only between one and 3.5 weeks after infusion of ibotenic acid.

Due to limitations, mainly for ethical reasons, on the use of nonhuman primates in biomedical research, the present pilot experiment aimed at applying LSI on macaque monkeys was restricted to a single animal (acute experiment lasting about one month). As a consequence, no statistical data on LSI measurements could be provided. Based on the present pilot LSI data, the next step may be to develop a LSI chronic recording site from the cortical surface (below a transparent artificial dura, as previously reported ${ }^{55-58}$ ), including the lesion site (for instance M1) as well as adjacent cortical areas possibly contributing to the functional recovery (e.g., the premotor cortex). This approach may replace the monitoring of the cortical lesion based on MRI, in case the latter facility is not accessible for nonhuman primates, with the restriction however that LSI is invasive (chronic recording chamber). As LSI and MRI provides parallel data with respect to the time course of changes of the cortical lesion, if available, MRI may be preferred as it is noninvasive. Moreover, the present study gives an approximation on how much the size of the cortical lesion assessed by MRI during the few weeks post-lesion overestimates the lesion extent observable post-mortem on histological sections.

\section{Conclusion}

Using in-parallel morphological (MRI) and functional imaging (LSI) methods in a macaque monkey, the present study shows that a restricted excitotoxic lesion of the motor cortex in nonhuman primate leads to a marked hypersignal in parallel to a dramatic increase in blood flow, at least up to a week postlesion. Traces of the lesion were still detectable using LSI after several weeks (about three to four weeks) in the form of a diminished cerebral blood flow as compared to pre-lesion, a time point at which the MRI signal had already returned to baseline. The presence of the lesion on a stable and long-term basis was corroborated by the histological post-mortem analysis.

\section{Acknowledgments}

The authors wish to thank the technical assistance of Dr. J. F. Brunet, Véronique Moret, Christine Roulin, Françoise Tinguely, and Christiane Marti (histology); Josef Corpataux, Laurent Bossy, Bernard Bapst and Bernard Morandi (animal house keeping); André Gaillard (mechanics), Bernard Aebischer (electronics), Laurent Monney (informatics), and Joël Baechler (MRI technician). We are grateful to Professor Bruno Weber and Dr. Fabrizio Croccolo for important discussions and help. This research was supported by the Swiss National Science Foundation [Grant Nos. 310000-110005 and 31003A-132465 to EMR, the National Centre of Competence in Research (NCCR) on "Neural plasticity and repair" (EMR), No. 2000020 - 126772 and 200020-117762 to FS]. 


\section{References}

1. G. Courtine, M. B. Bunge, J. W. Fawcett, R. G. Grossman, J. H. Kaas, R. Lemon, I. Maier, J. Martin, R. J. Nudo, A. Ramon-Cueto, E. M Rouiller, L. Schnell, T. Wannier, M. E. Schwab, and V. R. Edgerton, "Can experiments in nonhuman primates expedite the translation of treatments for spinal cord injury in humans?," Nat. Med. 13, 561-566 (2007).

2. J. P. Capitanio and M. E. Emborg, "Contributions of non-human primates to neuroscience research," Lancet 371, 1126-1135 (2008).

3. R. N. Lemon, "Descending pathways in motor control," Аnnu. Rev. Neurosci. 31, 195-218 (2008).

4. Y. Liu and E. M. Rouiller, "Mechanisms of recovery of dexterity following unilateral lesion of the sensorimotor cortex in adult monkeys," Exp. Brain Res. 128, 149-159 (1999).

5. E. Schmidlin, T. Wannier, J. Bloch, and E. M. Rouiller, "Progressive plastic changes in the hand representation of the primary motor cortex parallel incomplete recovery from a unilateral section of the corticospinal tract at cervical level in monkeys," Brain Res. 1017, 172-183 (2004).

6. E. Schmidlin, T. Wannier, J. Bloch, A. Belhaj-Saif, A. Wyss, and E. M. Rouiller, "Reduction of the hand representation in the ipsilateral primary motor cortex following unilateral section of the corticospinal tract at cervical level in monkeys," BMC Neurosci. 6, 56 (2005).

7. T. Wannier, E. Schmidlin, J. Bloch, and E. M. Rouiller, “A unilateral section of the corticospinal tract at cervical level in primates does not lead to measurable cell loss in motor cortex," J. Neurotrauma 22, 703 717 (2005).

8. P. Freund, E. Schmidlin, T. Wannier, J. Bloch, A. Mir, M. E. Schwab, and E. M. Rouiller, "Nogo-A-specific antibody treatment enhances sprouting and functional recovery after cervical lesion in adult primates," Nat. Med. 12, 790-792 (2006).

9. P. Freund, T. Wannier, E. Schmidlin, J. Bloch, A. Mir, M. E. Schwab, and E. M. Rouiller, "Anti-Nogo-A antibody treatment enhances sprouting of corticospinal axons rostral to a unilateral cervical spinal cord lesion in adult macaque monkey," J. Comp. Neurol. 502, 644-659 (2007).

10. P. Freund, E. Schmidlin, T. Wannier, J. Bloch, A. Mir, M. E. Schwab, and E. M. Rouiller, "Anti-Nogo-A antibody treatment promotes recovery of manual dexterity after unilateral cervical lesion in adult primatesre-examination and extension of behavioral data," Eur. J. Neurosci. 29 983-996 (2009).

11. M. Kaeser, A. F. Wyss, S. Bashir, A. Hamadjida, Y. Liu, J. Bloch, J. F. Brunet, A. Belhaj-Saif, and E. M. Rouiller, "Effects of unilateral motor cortex lesion on ipsilesional hand's reach and grasp performance in monkeys: relationship with recovery in the contralesional hand," $J$. Neurophysiol. 103, 1630-1645 (2010).

12. M. Kaeser, J. F. Brunet, A. F. Wyss, A. Belhaj-Saif, Y. Liu, A. Hamadjida, E. M. Rouiller, and J. Bloch, "Autologous adult cortical cell transplantation enhances functional recovery following unilateral lesion of motor cortex in primates: a pilot study," Neurosurg. 68, 1405-1417 (2011).

13. S. B. Frost, S. Barbay, K. M. Friel, E. J. Plautz, and R. J. Nudo, "Reorganization of remote cortical regions after ischemic brain injury: a potential substrate for stroke recovery," J. Neurophysiol. 89, 3205-3214 (2003).

14. N. Dancause, S. Barbay, S. B. Frost, E. J. Plautz, D. F. Chen, E. V. Zoubina, A. M. Stowe, and R. J. Nudo, "Extensive cortical rewiring after brain injury," J. Neurosci. 25, 10167-10179 (2005).

15. N. Dancause, S. Barbay, S. B. Frost, E. V. Zoubina, E. J. Plautz, J. D. Mahnken, and R. J. Nudo, "Effects of small ischemic lesions in the primary motor cortex on neurophysiological organization in ventral premotor cortex," J. Neurophysiol. 96, 3506-3511 (2006).

16. Y. Murata, N. Higo, T. Oishi, A. Yamashita, K. Matsuda, M. Hayashi, and S. Yamane, "Effects of motor training on the recovery of manual dexterity after primary motor cortex lesion in macaque monkeys," $J$. Neurophysiol. 99, 773-786 (2008).

17. D. G. Darling, M. A. Pizzimenti, D. L. Rotella, C. R. Peterson, S. M. Hynes, J. Ge, K. Solon, D. W. McNeal, K. S. Stilwell-Morecraft, and R. J. Morecraft, "Volumetric effects of motor cortex injury on recovery of dexterous movements," Exp. Neurol. 220, 90-108 (2009).

18. A. F. Fercher and J. D. Briers, "Flow visualization by means of single exposure speckle photography," Opt. Commun. 37, 326-330 (1981).
19. J. D. Briers, "Laser Doppler, speckle and related techniques for blood perfusion mapping and imaging," Physiol, Meas 22(4), R35-R66 (2001).

20. A. K. Dunn, A. Devor, H. Bolay, M. L. Andermann, M. A. Moskowitz, A. M. Dale, and D. A. Boas, "Simultaneous imaging of total cerebral hemoglobin concentration, oxygenation, and blood flow during functional activation," Opt. Lett. 28, 28-30 (2003).

21. A. K. Dunn, H. Bolay, M. A. Moskowitz, and D. A. Boas, "Dynamic imaging of cerebral blood flow using laser speckle," J. Cereb. Blood Flow Metab. 21,195-201 (2001).

22. C. Ayata, A. K. Dunn, Y. Gursoy-Ozdemir, Z. Huang, D. A. Boas, and M. A. Moskowitz, "Laser speckle flowmetry for the study of cerebrovascular physiology in normal and ischemic mouse cortex," J Cereb. Blood Flow Metab. 24, 744-755 (2004).

23. T. Durduran, M. G. Burnett, G. Yum, C. Zhoum, D. Furuyam, A. G. Yodh, J. A. Detrem, and J. H. Greenbergm, "Spatiotemporal quantification of cerebral blood flow during functional activation in rat somatosensory cortex using laser-speckle flowmetry," J. Cereb. Blood Flow Metab. 24, 518-525 (2004).

24. B. Weber, C. Burger, M. T. Wyss, G. K. von Schulthess, F. Scheffold, and A. Buck, "Optical imaging of the spatiotemporal dynamics of cerebral blood flow and oxidative metabolism in the rat barrel cortex," Eur. J. Neurosci. 20, 2664-2670 (2004).

25. J. S. Paul, A. R. Luft, E. Yew, and F. S. Sheu, "Imaging the development of an ischemic core following photochemically induced cortical infarction in rats using laser speckle contrast analysis (LASCA)," Neuroimage 29, 38-45 (2006).

26. J. S. Paul, S. Fwu-Shan, and A. R. Luft, "Early adaptation in somatosensory cortex after focal ischemic injury to motor cortex," Exp. Brain Res. 168, 178-185 (2006).

27. Z. Wang, P. Li, W. Luo, S. Chen, and Q. Luo, "Peri-infarct temporal changes in intrinsic optical signal during spreading depression in focal ischemic rat cortex," Neurosci. Lett. 424, 133-138 (2007).

28. W. Luo, Z. Wang, P. Li, S. Zeng, and Q. Luo, "A modified mini-stroke model with region-directed reperfusion in rat cortex," J. Cereb. Blood Flow Metab. 28, 973-983 (2008).

29. J. Luckl, C. Thou, T. Durduran, A. G. Yodh, and J. H. Greenberg, "Characterization of periinfarct flow transients with laser speckle and doppler after middle cerebral artery occlusion in the rat," J. Neurosci. Res. 87, 1219-1229 (2009).

30. G. A. Armitage, K. G. Todd, A. Shuaib, and I. R. Winship, "Laser speckle contrast imaging of collateral blood flow during acute ischemic stroke," J. Cereb. Blood Flow Metab. 30, 1432-1436 (2010)

31. W. J. Tom, A. Ponticorvo, and A. K. Dunn, "Efficient processing of laser speckle contrast images," IEEE Trans. Med. Imaging 27, 1728-1738 (2008).

32. P. Zakharov, A. Völker, A. Buck, B. Weber, and F. Scheffold, "Quantitative modeling of laser speckle imaging," Opt. Lett. 31, 3465-3467 (2006).

33. P. Zakharov, A. C. Völker, M. T. Wyss, F. Haiss, N. Calcinaghi, C. Zunzunegui, A. Buck, F. Scheffold, and B. Weber, "Dynamic laser speckle imaging of cerebral blood flow," Opt. Express 17, 13904-13917 (2009).

34. A. C. Völker, P. Zakharov, B. Weber, A. Buck, and F. Scheffold, "Laser speckle imaging with an active noise reduction scheme," Opt. Express 13, 9782-9787 (2005).

35. A. B. Parthasarathy, W. J. Tom, A. Gopal, Zhang, and A. K. Dunn, "Robust flow measurement with multi-exposure speckle imaging," Opt Express 16, 1975-1989 (2008).

36. P. C. Li, S. Ni, L. Zhang, S. Zeng, and Q. Luo, "Imaging cerebral blood flow through the intact rat skull with temporal laser speckle imaging," Opt. Lett. 31, 1824-1826 (2006).

37. N. Hecht, J. Woitzik, J. P. Dreier, and P. Vajkoczy, "Intraoperative monitoring of cerebral blood flow by laser speckle contrast analysis," Neurosurg. Focus 27(4), E11 (2009).

38. H. Cheng, Q. Luo, S. Zeng, S. Chen, J. Cen, and H. Gong, "Modified laser speckle imaging method with improved spatial resolution," $J$. Biomed. Opt. 8, 559-564 (2003).

39. M. L. Beaud, E. Schmidlin, T. Wannier, P. Freund, J. Bloch, A. Mir, M. E. Schwab, and E. M. Rouiller, "Anti-Nogo-A antibody treatment does not prevent cell body shrinkage in the motor cortex in adult monkeys subjected to unilateral cervical cord lesion," BMC Neurosci. 9, 5 (2008). 
Peuser et al.: Follow-up of cortical activity and structure after lesion with laser speckle imaging...

40. J. Bloch, M. Kaeser, Y. Sadeghi, E. M. Rouiller, D. E. Redmond Jr., and J. F. Brunet, "Doublecortin-positive cells in the adult primate cerebral cortex and possible role in brain plasticity and development," J. Comp. Neurol. 519, 775-789 (2011).

41. P. Leroux, C. Hennebert, J. Cateau, N. Legros, O. Hennebert, V. Laudenbach, and S. Marret, "Neuroprotective effects vary across nonsteroidal anti-inflammatory drugs in a mouse model of developing excitotoxic brain injury," Neuroscience 167, 716-723 (2010).

42. Y. Ozsunar, P. E. Grant, T. A. G. M. Huisman, P. W. Schaefer, O. Wu, A. G. Sorensen, W. J. Koroshetz, and R. G. Gonzalez, "Evolution of water diffusion and anisotropy in hyperacute stroke: significant correlation between fractional anisotropy and T2," AJNR Am. J. Neuroradiol. 25, 699-705 (2004).

43. M. Thurnher, "Brain Ischemia - Imaging in acute stroke," The Radiology Assistant, www.radiologyassistant.nl/en/483910a4b6f14 (2008).

44. A. Ritzl, S. Meisel, H. J. Wittsack, G. R. Fink, M. Stiebler, U. Mödder, and R. J. Seitz, "Development of brain infarct volume as assessed by magnetic resonance imaging (MRI): follow-up of diffusion-weighted MRI lesions," J. Magn. Reson. Imaging 20, 201-207 (2004).

45. J. L. Sunshine, R. W. Tarr, C. F. Lanzieri, D. M. D. Landis, W. R. Selman, and J. S. Lewin, " Hyperacute stroke: ultrafast MR imaging to triage patients prior therapy," Radiology 212, 325-332 (1999).

46. A. E. Baird and S. Warach, "Magnetic resonance imaging of acute stroke," J. Cereb. Blood Flow. Metab. 18, 583-609 (1998).

47. P. Lavenex, P. Banta Lavenex, and D. G. Amaral, "Spatial relational learning persists following neonatal hippocampal lesions in macaque monkeys," Nat. Neurosci. 10, 234-239 (2007).

48. L. Malkova, C. K. Lex, M. Mishkin, and R. C. Saunders, "MRI-based evaluation of locus and extent of neurotoxic lesions in monkeys," Hippocampus 11, 361-370 (2001).
49. N. Ben-Horin, S. Hazvi, P. Bendel, and R. Schul, "The ontogeny of a neurotoxic lesion in rat brain revealed by combined MRI and histology," Brain Res. 718, 97-104 (1996).

50. J. John, V. Govindaraju, P. Raghunathan, and V. M. Kumar, "Magnetic resonance imaging of temporal changes of neurotoxic lesion in the rat," Brain Res. Bull. 40, 273-277 (1996).

51. N. Shemesh, O. Sadan, E. Melamed, D. Offen, and Y. Cohen, "Longitudinal MRI and MRSI characterization of the quinolinic acid rat model for excitotoxicity: peculiar apparent diffusion coefficients and recovery of N-acetyl aspartate levels," NMR Biomed. 23, 196-206 (2010).

52. W. T. Newsome, R. H. Wurtz, M. R. Dürsteler, and A. Mikami, "Deficits in visual motion processing following ibotenic acid lesions of the middle temporal visual area of the macaque monkey," J. Neurosci. 5, 825-840 (1985).

53. W. H. Merigan, T. A. Nealey, and J. H. Maunsell, "Visual effects of lesions of cortical area V2 in macaques," J. Neurosci. 13, 3180-3191 (1993).

54. K. Rudolf and T. Pasternak, "Transient and permanent deficits in motion perception after lesions of cortical areas MT and MST in the macaque monkey," Cereb. Cortex 9, 90-100 (1999).

55. E. Shtoyerman, A. Arieli, H. Slovin, I. Vanzetta, and A. Grinvald, "Long-term optical imaging and spectroscopy reveal mechanisms underlying the intrinsic signal and stability of cortical maps in V1 of behaving monkeys," J. Neurosci. 20, 8111-8121 (2000).

56. A. Arieli, A. Grinvald, and H. Slovin, "Dural substitute for long-term imaging of cortical activity in behaving monkeys and its clinical implications," J. Neurosci. Methods 114,119-133 (2002).

57. L. M. Chen, B. Heider, G. V. Williams, F. L. Healy, B. M. Ramsden, and A. W. Roe, "A chamber and artificial dura method for long-term optical imaging in the monkey," J. Neurosci. Methods 113, 41-49 (2002).

58. B. Heider, G. Jando, and R. M. Siegel, "Functional architecture of retinotopy in visual association cortex of behaving monkeys," Cereb. Cortex 15, 460-478 (2005). 